\title{
Clinical Evaluation of UR-System 2 for Recovery of Motor Function of Plegic Upper Limb after Stroke
}

\author{
Hirofumi Tanabe, Masahiro Mitsukane \\ Department of Health Sciences, Shonan University of Medical Sciences \\ 16-48 Kamishinano, Totsuka-ku, Yokohama, Kanagawa 244-0806, Japan \\ E-mail: hirofumi.tanabe@sums.ac.jp \\ Norihiro Toya, Ryosuke Takeichi, Hitomi Hattori, Yoshifumi Morita \\ Nagoya Institute of Technology \\ Gokiso-cho, Showa-ku, Nagoya, Aichi 466-8555, Japan \\ Yoshiaki Takagi, Norio Hasegawa \\ Sanyo Machine Works, Ltd. \\ 1 Oka, Okimura, Kitanagoya-shi, Aichi 481-8540, Japan
}

\begin{abstract}
We developed a new training system UR-System 2 for restoring motor function of the upper limb after stroke in patients with hemiplegia. And then, we conducted clinical evaluation of the therapeutic effect of training with the UR-System 2 in six patients. The UR-System uses Proprioceptive Neuromuscular Facilitation (PNF) to promote muscle strength. The results show the immediate therapeutic effect of training with the UR-System 2 for restoring the motor function of the upper limb.
\end{abstract}

Keywords: Rehabilitation, Training system, Hemiplegic stroke patient, Clinical evaluation, PNF

\section{Introduction}

The synergy movement pattern often seen in hemiplegic patients after stroke is one of the factors inhibiting improvement of motor function. According to the Brunnstrom recovery stages of hemiplegia, elbow flexion, pronation/supination of the forearm, wrist flexion, and finger flexion occur abnormally and simultaneously in hemiplegic patients during the early stage of recovery; this is called the synergy movement pattern. In the recovery stage, a patient gradually evolves from a synergy movement pattern, and will be able to extend the elbow, supinate the forearm, and extend the wrist and fingers ${ }^{1}$. Proprioceptive Neuromuscular Facilitation (PNF) is an effective therapy facilitating recovery from spastic paralysis ${ }^{2,3}$. Since this repetitive technique is performed manually, the therapist's physical workload is considerable. Therefore, we are developing a training system for recovery of normal movement isolated from synergy movement patterns by facilitating contraction of elbow extension and forearm supination muscles.

In our previous work $^{4}$, we developed a training system (UR-System: Useful and Ultimate Rehabilitation System) for recovery of motor function of the upper limb after stroke in patients with hemiplegia. This system uses PNF to promote muscle strength. Clinical evaluation of the therapeutic effect of training with the UR-System was performed in eight patients for two weeks. Active ranges of motion (A-ROMs) of elbow extension and forearm supination improved after training with the UR-System. Moreover, the modified Ashworth scale (MAS) scores ${ }^{5}$ for elbow extension and forearm supination increased. This means that spastic 
paralysis was reduced. This shows the effectiveness of training with the UR-System for recovery of motor function of the upper limb.

However, the following problems persist. Since the UR-System was not equipped with sensors that can measure the movement of the paralyzed forearm and the force/torque exerted by the forearm, the therapeutic effect was not evaluated in detail (Problem I). It was difficult for therapists to adjust the parameters of the rubber belt to facilitate elbow extension suitable for the patient's condition (Problem II).

In order to solve these problems this study developed a new training system, the UR-System 2. We evaluated the immediate therapeutic effect of recovery of motor function by training with the UR-System 2 .

\section{UR-System 2}

To solve Problem I, we introduced sensors on the brace. To solve Problem II, we developed an elbow-pushing arm on the brace in place of the rubber belt to facilitate elbow extension. Moreover, since the previous URSystem was over-engineered, we reconsidered the specifications, and developed the UR-System 2. As a result, the number of parts, weight of the mechanical system, and total cost decreased.

The UR-System 2 is a force display system with one degree of freedom. The system consists of a mechanical system and a controller. The mechanical system consists of a training arm, a powder brake (SINFONIA TECHNOLOGY CO., LTD., PRB-2.5H), and a brace. The brace was used to secure the patient's forearm to the training system. The patient moves the training arm independently. The powder brake generates a resistance force during training. Four different resistance patterns, i.e., step, slope, wall, and constant modes, were installed in the controller. The length of the training arm can be altered within the range of $0.60-0.89 \mathrm{~m}$. The maximum resistance is $49 \mathrm{~N}$ when the length of the training arm is $0.75 \mathrm{~m}$

The brace has three degrees of freedom, allowing rolling, yawing, and pitching motions of the fixing plate. The rolling motion can be switched between free motion and fixed motion with a double nut. When the UR-System 2 is used in training, the rolling motion is fixed, but is allowed when evaluating the therapeutic effect. The brace is equipped with three potentiometers
(ALPS ELECTRIC CO. LTD., RDC803001A) and a 6axis force sensor (Leptrino Co. Ltd., PFS055YA251A6). The potentiometers measure roll, yaw, and pitch angles of the fixing plate of the brace. The 6-axis force sensor measures the force and torque between the forearm and the fixing plate. An elbowpushing arm is installed on the fixing plate to facilitate elbow extension. As the patient moves the forearm forward, the elbow-pushing arm pushes the elbow to facilitate contraction of a deltoid muscle. The elbowpushing arm is rigid and does not bend to the external force. The contact portion of the elbow-passing arm to the deltoid muscle is made by harder cushion. When the forearm is fixed to the brace, the portions of the forearm near the wrist and the elbow are fixed with a nonexpandable bandage and an expandable bandage, respectively. Therefore, the portion of the forearm near the elbow can leave the brace due to the extensibility of the expandable bandage. Moreover, the patient can extend his/her elbow fully in spite of the existence of the elbow pushing arm. If a non-expandable bandage is not used, the patient cannot extend his/her elbow fully.

Because this system is not equipped with motors, it is extremely safe and economical. It should be noted that the system is used only for active exercise training.

The UR-System 2 was equipped with various functions. The resistance display function allows therapists to perform various types of resistance training by changing the arm length and the resistance level. The touch panel parameter setting function allows the parameters of the resistance patterns to be easily set by pushing buttons on the touch panel display. The parameters consist of the magnitudes and positions of the resistance patterns. The magnitudes are selected from among twenty-five levels. The positions are determined by moving the training arm to the desired position. This function provides good visibility and easy operability for the therapist.

The resistance to the movement of the forearm and compression of the elbow are expected to facilitate elbow extension. These concepts are based on facilitation elements, namely resistance to movement, compression arthrodesis, passive movement, and manual contact in PNF techniques. Therefore, the patient is expected to extend the elbow joint fully. Moreover, pronation and supination of the forearm, 
wrist flexion, and finger flexion are not permitted during elbow extension and flexion, because the forearm is fixed on the fixing plate of the brace, preventing a synergy movement pattern from occurring. Therefore, isolated movement should be facilitated by using the UR-System 2.

\section{Clinical Evaluation of Therapeutic Effect}

\subsection{Method}

We evaluated six patients who satisfied the following conditions:

1) The subject had a hemiplegic upper limb after a stroke.

2) More than one year had passed since stroke onset.

3) The subject did not have pain in training with the UR-System 2.

4) The Brunnstrom Stage was IV or V.

The post-stroke durations were 10 years in Subject A, 12 in Subject B, two in Subject C, four in Subject D, one and one-half in Subject E, and five in Subject F. We obtained written consent from all patients.

We used two training protocols for a comparative study. One employed training without PNF-facilitating elements, namely resistance and compression arthrodesis, and was referred to as Control-training. The other employed training with PNF-facilitating elements, and was referred to as PNF-training. PNF-training was performed at least three days after Control-training.

The patient performed repeated training for elbow flexion and extension with the paralyzed forearm in the supinated position. Repeated training was performed 50 times per set for five sets. The rest time between sets was 5 minutes.

The therapeutic effects of training with the URSystem 2 were assessed using the MAS and the A-ROM test before and after training, and were compared with those of Control-training and PNF-training.

\subsection{Results and discussion}

All subjects completed two types of training with the UR-System 2. Table 1 presents the summary of scores for outcome measures. Table 1 shows that the A-ROMs and MAS score in PNF-Training increased significantly after training. Figures 2-4 show the A-ROMs and MAS score were improved greatly in PNF-Training as compared with Control-training. Thus, isolated movement was facilitated by PNF-training with the URSystem 2. The immediate effect of restoring motor function in PNF-training was excellent as compared with Control-training. Moreover, it is noteworthy that motor function was restored even in Patients A and B, who had stroke episodes more than ten years prior to training. Consequently, training with the UR-System 2 can immediately improve motor function of the upper limb after stroke in patients with hemiplegia.

Since the therapeutic effect shown in this study is immediate, we will investigate this effect after daily continuous PNF-training. Moreover, the workload of therapists will decrease because the system can be used for independent training for recovery of motor function.

\section{Conclusion}

The immediate therapeutic effect of PNF-training with the UR-System 2 was shown from the results of the clinical evaluation. PNF-training with the system can immediately improve motor function of the hemiplegic upper limb.

In a future study, we will confirm the therapeutic effect after daily continuous PNF-training with the system 2 for a larger number of subjects, and develop a training method for recovery of motor function of the shoulder and lower extremities of hemiplegic patients by modifying the UR-System 2 .

\section{References}

1. I. Safaz, B. Ylmaz, E. Yaşar, R. Alaca, Brunnstrom recovery stage and motricity index for the evaluation of upper extremity in stroke: Analysis for correlation and responsiveness, Int. J. Rehabilitation Res., 32(3) (2009), pp. 228-231.

2. J. Stein, R. Harvey, C. Winstein, G. Wittenberg, R Zorowitz, Stroke recovery and rehabilitation, Demos Medical Publishing, (NY,2008).

3. K. B. Hindle, T. J. Whitcomb, W. O. Briggs, J. Hong, Proprioceptive neuromuscular facilitation (PNF): Its mechanisms and effects on range of motion and muscular function, J. Hum. Kinet., 31 (2012), pp. 105-113.

4. Y. Morita, N. Sato, H. Ukai, H. Tanabe, T. Nagao, R. Tanemura, Y. Takagi, Y. Aoki, Clinical evaluation of training system for recovery of motor function after stroke in patients with hemiplegia, Proc. of the 2nd Int. Conf. on NeuroRehabilitation (ICNR2014) (2014), pp. 83-92.

5. Ashworth B, Preliminary trial of carisoprodal in multiple sclerosis, Practitioner, 192 (1964), pp.540-542. 


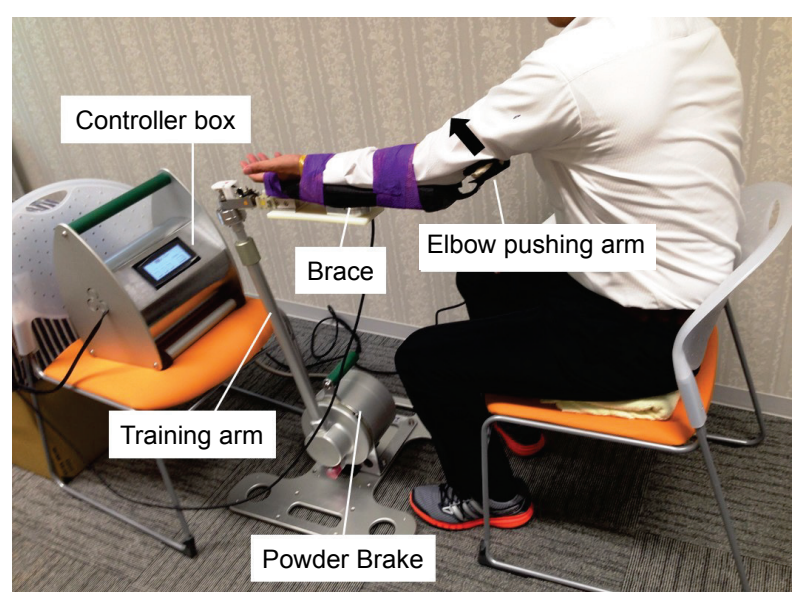

Fig. 1. UR-System 2.

Table 1. Summary of scores for outcome measures (Control: Control-training, PNF: PNF-training,

Pre: Before training, Post: After training,

Diff: Difference between the results before and after training,

Positive difference values mean improvement.)

(a) A-ROM of elbow extension.

\begin{tabular}{ccccccc}
\hline \multirow{2}{*}{ Subject } & \multicolumn{3}{c}{ Control } & \multicolumn{3}{c}{ PNF } \\
\cline { 2 - 7 } & Pre & Post & Diff. & Pre & Post & Diff \\
\hline A & -20 & -15 & +5 & -20 & -5 & +15 \\
\hline B & -15 & -25 & -10 & -15 & -5 & +10 \\
\hline C & -25 & -25 & +0 & -25 & -10 & +15 \\
\hline D & -20 & -15 & +5 & -20 & 0 & +20 \\
\hline E & -20 & -15 & +5 & -20 & -10 & +10 \\
\hline F & -30 & -15 & +5 & -30 & -5 & +25
\end{tabular}

(b) A-ROM of supination of forearm.

\begin{tabular}{ccccccc}
\hline \multirow{2}{*}{ Subject } & \multicolumn{3}{c}{ Control } & \multicolumn{3}{c}{ PNF } \\
\cline { 2 - 7 } & Pre & Post & Diff. & Pre & Post & Diff. \\
\hline A & 65 & 75 & +10 & 65 & 90 & +25 \\
\hline B & 40 & 55 & +15 & 40 & 70 & +30 \\
\hline C & 55 & 60 & +5 & 55 & 65 & +10 \\
\hline D & 35 & 45 & +10 & 35 & 55 & +20 \\
\hline E & 40 & 65 & +25 & 40 & 70 & +30 \\
\hline F & 65 & 75 & +10 & 65 & 90 & +25 \\
\hline
\end{tabular}

(c) MAS Score of elbow flexor group.

\begin{tabular}{ccccccc}
\hline \multirow{2}{*}{ Subject } & \multicolumn{3}{c}{ Control } & \multicolumn{3}{c}{ PNF } \\
\cline { 2 - 8 } & Pre & Post & Diff. & Pre & Post & Diff. \\
\hline A & 4 & 4 & 0 & 4 & 5 & +1 \\
\hline B & 4 & 4 & 0 & 4 & 5 & +1 \\
\hline C & 3 & 4 & +1 & 3 & 4 & +1 \\
\hline D & 3 & 3 & 0 & 3 & 4 & +1 \\
\hline E & 3 & 4 & +1 & 3 & 4 & +1 \\
\hline F & 3 & 3 & 0 & 3 & 4 & +1 \\
\hline
\end{tabular}

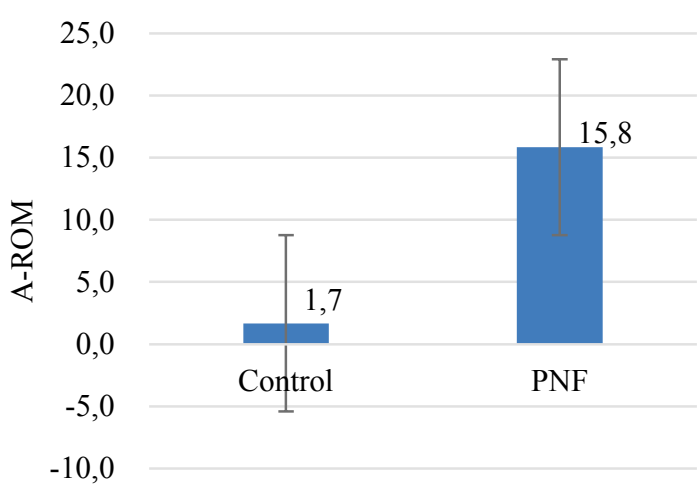

Fig. 2. Improvement of A-ROM of elbow extension.

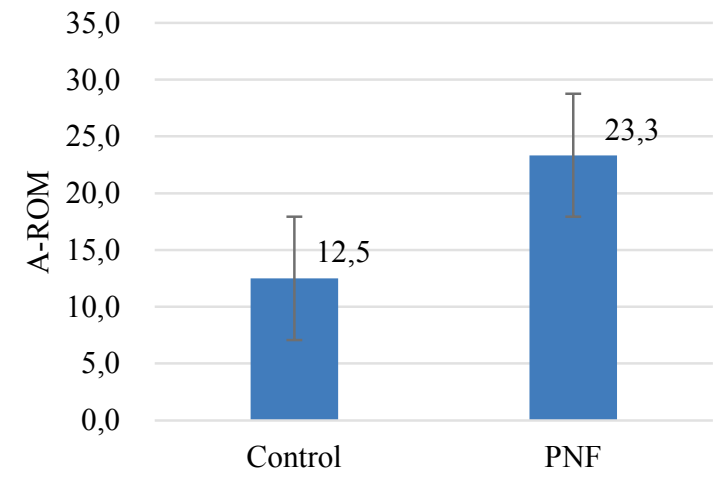

Fig. 3. Improvement of A-ROM of supination of forearm.

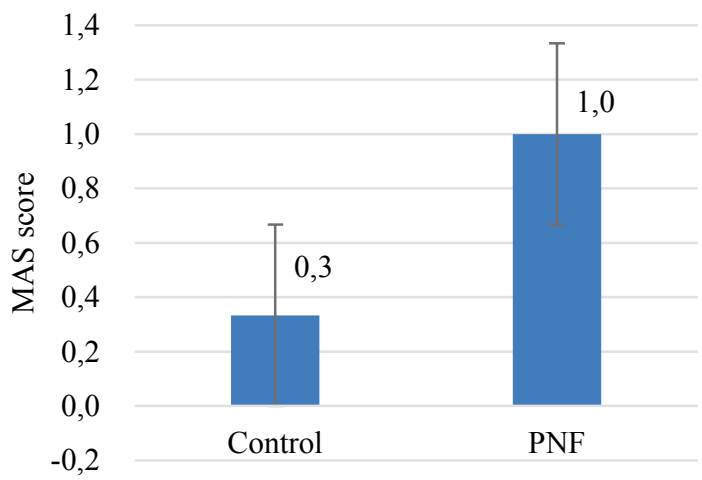

Fig. 4. Improvement of MAS Score of elbow flexor group. 